\title{
A European Network for the Investigation of Gender Incongruence: Endocrine Part
}

\author{
M. J. H. J. Dekker, MD, PhD, K. Wierckx, MD, PhD, ${ }^{2}$ E. Van Caenegem, MD, PhD, ${ }^{2}$ M. Klaver, MD, \\ B. P. Kreukels, $\mathrm{PhD}^{3}$ E. Elaut, $\mathrm{PhD}^{4}$ A. D. Fisher, $\mathrm{MD}, \mathrm{PhD}_{,}{ }^{5}$ M. A. A. van Trotsenburg, $\mathrm{MD},{ }^{6}$ T. Schreiner, $\mathrm{MD},{ }^{7}$ \\ M. den Heijer, MD, PhD, and G. T'Sjoen, MD, PhD ${ }^{2,4}$
}

\section{ABSTRACT}

Introduction: Cross-sex hormone therapy is an essential part of gender affirming treatment of transgender individuals. Studies systematically describing the physical and psychological effects of hormonal treatment of transgender persons are scarce.

Aim: The aim of the current protocol is to evaluate clinical and side-effects of cross-sex hormonal treatment in trans persons.

Methods: The European Network for the Investigation of Gender Incongruence (ENIGI) is a multicenter prospective study. Because of the relatively low prevalence of the condition and small number of specialized centers, international collaboration is warranted. Four European treatment centers, Ghent, Oslo, Florence, and Amsterdam, developed a common study and treatment protocol.

Main Outcome Measures: Outcome measures include hormonal and metabolic parameters, bone density, secondary sex and anthropometric characteristics, and physical and psychological well-being.

Results: Thus far, 333 trans women and 343 trans men have been included in the ENIGI Endocrine protocol. The study is still ongoing.

Conclusion: In recent years, the number of trans persons seeking gender affirming treatment has increased. However, well-designed prospective studies evaluating safety and effectiveness of current hormonal treatment protocols are lacking. Therefore we started the ENIGI collaboration. In this article we give a detailed description of the study protocol, objectives, and design of the ENIGI Endocrine protocol.

J Sex Med 2016;13:994-999. Copyright $@$ 2016, International Society for Sexual Medicine. Published by Elsevier Inc. All rights reserved.

Key Words: Gender Dysphoria; Cross-Sex Hormonal Treatment; Prospective Cohort Study

\section{INTRODUCTION}

"Gender dysphoria" (GD) refers to the distress related to an incongruence between one's experienced gender and one's

Received November 2, 2015. Accepted March 28, 2016.

'Department of Endocrinology, VU University Medical Center, Amsterdam, The Netherlands;

${ }^{2}$ Department of Endocrinology, Ghent University Hospital, Ghent, Belgium;

${ }^{3}$ Department of Medical Psychology, VU University Medical Center, Amsterdam, the Netherlands;

${ }^{4}$ Center for Sexology and Gender, Chent University Hospital, Ghent, Belgium;

${ }^{5}$ Sexual Medicine and Andrology Unit, Department of Experimental, Clinical and Biomedical Sciences, University of Florence, Florence, Italy;

${ }^{6}$ Department of Obstetrics and Gynaecology, VU University Medical Center;

${ }^{7}$ Department of Endocrinology, Oslo University Hospital, Oslo, Norway

Copyright (c) 2016, International Society for Sexual Medicine. Published by Elsevier Inc. All rights reserved.

http://dx.doi.org/10.1016/j.jsxm.2016.03.371 assigned gender that has been present for at least 6 months. ${ }^{1}$ This condition has a great negative impact on physical, social, and psychological well-being and a large proportion of trans persons (but not all) desire gender affirming treatment.

For most trans individuals, hormonal treatment is an essential part of their sex change. Harry Benjamin (January 12, 1885-August 24, 1986) born in Germany, was an American endocrinologist and sexologist who was one of the pioneers treating patients with cross-sex hormones around the middle of the twentieth century. Although gender reassignment was still a matter of much debate, over half a century later hormone therapy and surgical reassignment are accepted as the mainstay treatment for gender dysphoria. ${ }^{2}$

Currently, endocrine treatment regimens are not standardized and include various forms, applications, and dosages of estrogens, progestins and (anti) androgens. ${ }^{3}$ Thus far, no randomized intervention trials have been performed to determine the best treatment regimens. Furthermore, no large prospective studies have evaluated 
(side) effects of cross-sex hormone treatment (CHT) in a structured manner. The European Network for the Investigation of Gender Incongruence (ENIGI) collaboration creates a unique opportunity to perform the much-needed research in this field. The main objective of the study is to describe the clinical effects and side-effects of hormonal treatment in adults with GD. All participating centers will evaluate these clinical effects using the same standardized measurements and questionnaires.

Previously, Kreukels et al ${ }^{4}$ presented the diagnostic protocol and psychological assessments used in the ENIGI Mental Health protocol. The close collaboration between both mental health and endocrine specialists offers a unique possibility for translational and interdisciplinary research. Furthermore, the international and multicenter approach of this study facilitates collaboration and exchange between different European expert centers. Another major advantage of this collaboration is that we can include larger number of patients in our study. This is of specific importance, because although patient numbers are increasing, GD is still a relatively rare condition.

\section{AIMS}

The aims of the endocrine part of the ENIGI collaboration is to evaluate the effects of $\mathrm{CHT}$ on hormonal and metabolic parameters, bone density, secondary sex and anthropometric characteristics, and physical and psychological well-being of trans persons. In this article, we present the study design and data collection procedures of the ENIGI Endocrine protocol.

\section{SUBJECTS AND METHODS}

\section{Participating Centers}

The ENIGI study is a multicenter prospective cohort study. So far, 4 West European gender identity clinics are participating in this collaboration. They include Ghent University Hospital, Belgium; VU University Medical Center in Amsterdam, the Netherlands; Rikshospitalet in Oslo, Norway; and University Hospital in Florence, Italy. These centers all use the ENIGI Mental Health protocol. The first trans persons were included in the ENIGI Endocrine protocol in 2010. Data presented in this article represent an update of our inclusions until March 12, 2015. At that time the first conference of the European Professional Association for Transgender Health (EPATH) was organized in Ghent, Belgium. Inclusion of trans persons is still ongoing in all participating centers.

The overall study protocol was approved by the Ethical Committee of Ghent University Hospital, Belgium. Every participating clinical center also obtained approval of their local ethical committees.

\section{Study Participants}

All patients underwent a standardized diagnostic procedure to confirm the diagnosis GD/gender incongruence and assess eligibility for treatment. The procedure of the ENIGI Mental Health protocol is described in Kreukels et al. ${ }^{4}$ In short, the mean duration of the diagnostic phase varies between 6 and 12 months and consists of regular meetings with a psychologist and/ or a psychiatrist. Once the diagnosis has been confirmed and there are no physical, psychological or social contraindications, hormonal treatment is started. In all centers, psychological counselling is offered during this phase, which is also called the "Social Transition Phase." During this 12- to 18-month period, persons experience life in the desired gender role on a daily basis. Once persons successfully go through this phase and there are no somatic contraindications, they are referred to the surgeon if they desire gender affirming surgery. All included subjects are above 16 years (Ghent and Oslo), above 17 years (Amsterdam), or above 18 years (Florence).

People are included in the ENIGI Endocrine protocol when they start medical treatment for GD/gender incongruence. Data on fulfillment of diagnostic criteria of current manuals for classification is collected in the ENIGI Mental Health protocol. Patients are eligible to participate if they have not used cross-sex hormones before and if they have sufficient knowledge of the native languages: Dutch or French for participants from Ghent, and Dutch, Italian, and Norwegian for patients from Amsterdam, Florence, and Oslo, respectively. At the start of CHT, patients receive oral and written information of the ENIGI endocrine protocol by their physician and written informed consent is obtained according to the institutional guidelines.

\section{Treatment Protocol}

\section{Trans women}

Cyproterone acetate in a once daily dose of $50 \mathrm{mg}$ is started in combination with an estradiol agent. In Ghent, Amsterdam, and Florence, estradiol valerate is prescribed $2 \mathrm{mg}$ twice daily, whereas in Oslo $4 \mathrm{mg}$ is given in 1 single dose. Although not well established, the ENIGI medical team felt that risk of thrombosis from estrogens was greater with oral agents than with transdermal preparations, perhaps due to the "first pass effect" of the liver. Therefore the protocol calls for transdermal estrogens for patients older than 45 years of age. In Amsterdam and Ghent we prescribe estradiol patches in a dose of $100 \mathrm{mcg} / 24$ hours. For some medical conditions, such as a history of thrombosis, treatment is started at a lower dose. In Florence patients can also choose from estradiol emidrate gel $1 \mathrm{mg}$ twice daily.

\section{Trans men}

As not all testosterone treatment regimens are reimbursed in the different European countries, treatment protocols differ between the clinical centers. In Ghent and Oslo, testosterone undecanoate $1000 \mathrm{mg}$ once per 12 weeks (Nebido) is prescribed. In the Netherlands, currently testosterone undecanoate injections are not covered by health insurance companies, so most patients choose between testosterone gel in a daily dose of $50 \mathrm{mg}$ or testosterone esters $250 \mathrm{mg}$ injections (Sustanon) every 2 
weeks. If they prefer, testosterone undecanoate injections are prescribed.

In Florence, costs for hormonal treatment are covered by The National Health Service only in some regions. Trans men can choose between testosterone gel in a daily dose of $50 \mathrm{mg}$ or different injectable formulations: testosterone undecanoate 1000 mg once per 12 weeks or testosterone enanthate $250 \mathrm{mg}$ (Testoenant $\left.^{(}\right)$injections every 2 weeks.

\section{Data Collection}

\section{Demographic characteristics}

At baseline (during the diagnostic phase) demographic characteristics are assessed. They include age, age of onset of GD, quality of life, education level (low: lower education and lower vocational; middle: secondary education, secondary vocational and high school; high: higher vocational, bachelor, master and $\mathrm{PhD}$ ), living situation, employment (no employment, full-time, part-time, and education), and sexual orientation.

\section{Clinical Measurements}

At baseline and during each visit, body weight and height are measured in light indoor clothing without shoes. Additionally waist, hip, and chest circumference are measured. The chest circumference is measured at the level of the nipples and just below the breasts, so cup size can be inferred. Blood pressure is measured using an electronic blood pressure monitor. Grip strength at the dominant hand is measured using an adjustable hand-held standard grip device (JAMAR hand dynamometer, Sammons and Preston; Bolingbrook, IL, USA).,

The Ferriman-Gallwey score is used to evaluate and quantify body hair in trans men. Because of shaving and local manipulation the Ferriman-Gallwey score is not meaningful in trans women. ${ }^{7}$ Acne is assessed using the Global Acne Grading Scale (GAGS). ${ }^{8}$ At baseline a detailed medical history is taken to evaluate contraindications for hormonal treatment. At each visit current smoking status, alcohol use, medication use, and intercurrent diseases are assessed.

At all visits all clinical measures are filled out on a standardized form. After the visit this information is entered in our database manually. Measures that are used for clinical purpose are also documented in our medical records and include length, weight, blood pressure, current smoking status and alcohol use, intercurrent disease, and medication use.

\section{Serum determinations}

Venous blood samples are obtained between $8 \mathrm{AM}$ and 10 AM after overnight fasting. Samples are analyzed at the local laboratory, as they are also used for clinical purposes. Serum measurements include haematocrit, alkaline phosphatase, gamma-glutamyltransferase, aspartate-aminotransferase, alanineaminotransferase, creatinine, glucose, total cholesterol, triglycerides, high-density lipoprotein cholesterol, low-density lipoprotein cholesterol, 25-hydroxycholecalciferol, calcium, albumin, testosterone, estradiol, sex hormone-binding globulin, luteinizing hormone, prolactin, and prostate-specific antigen (in trans women aged 45 years and older).

\section{Serum Bank}

Next to the serum samples drawn for clinical evaluation additional samples are stored for future research purposes. These samples are collected at baseline, after 3 months and 1, 2 and 10 years of CHT. Next to this, at baseline, buffy coats are stored for future DNA research. In the near future we will start by extracting DNA from these samples. All samples are stored at $-80^{\circ} \mathrm{C}$ at every local center.

\section{Bone Measurements and Body Composition}

Body fat and lean mass, bone mineral content, bone area, and areal bone mineral density at the lumbar spine and left proximal femur (total hip and femoral neck region) are measured using dual X-ray absorptiometry (DEXA). In Ghent additional bone measurements are available using peripheral quantitative computed tomography (pQCT). ${ }^{9}$ Bone measurements are performed at baseline, and after 1, 2, and 10 years of hormonal treatment.

\section{Questionnaires}

At each visit questionnaires are handed out by our secretary staff or physicians. It takes approximately 15 to 20 minutes to fill out these forms. The questionnaires are processed using TeleForm automated data extraction (Hewlett-Packard Company; Palo Alto, CA, USA).

Physical activity: Habitual physical activity is measured using the Baecke Activity Questionnaire, which consists of 16 questions classified into 3 different dimensions: work, sport, and nonsports leisure activity. Each domain can receive a score from 1 to 5 points, thus allowing a total score from 3 (minimum activity) to 15 (maximum activity). ${ }^{10}$

Experienced vocal performance: The Trans Voice Questionnaire is a 30 -item self-administered questionnaire that evaluates the psychosocial consequences of voice disorders and consists of 3 dimensions: functional, physical, and emotional impairment. A score of 0 is equivalent to no disability and a score of 120 is equivalent to maximum disability. ${ }^{11}$

Side-effects questionnaire: We constructed a questionnaire to evaluate side-effects of hormonal treatment such as psychovegetative symptoms, physical complaints, cognition, emotionality and sexuality, genital complaints, and pain.

The following 4 questionnaires are part of the Mental HealthEndocrinology protocol:

Positive Affect Negative Affect Scale (PANAS): The PANAS is a 20-item questionnaire that measures long-term changes in affect. Participants are asked to what extent they experience certain 
feelings such as anxiety, happiness, or guilt on a 5-point scale from "very little" to "very much". ${ }^{12}$

Aggression proneness: The STAXI-2 State Anger scale is a 15item questionnaire that evaluates aggression. Participants rank certain statements along a 4-point continuum from "not at all" to "very much." The questionnaire evaluates angry feelings on 3 subscales: feeling angry, feeling like expressing anger verbally, and feeling like expressing anger physically. ${ }^{13}$

Sexual desire: The Sexual Desire Inventory (SDI) is a selfadministered 14-item questionnaire that aims to measure sexual desire. The SDI measures the individual's thoughts as well as actual experiences. Fourteen questions assess the strength, frequency, and importance of an individual's desire for sexual activity with others and by themselves. The score ranges from 0 (no sexual desire) to 112 (maximum desire). ${ }^{14}$

Sexual orientation questionnaire: This short questionnaire consists of 4 questions in the "background data interview" which is part of the ENIGI Mental Health protocol. These questions evaluate gender roles in sexual fantasy, and sexual orientation.

Psychological questionnaires: For a detailed description of the additional psychological questionnaires used in the ENIGI Mental Health protocol, see Kreukels et al. ${ }^{4}$ These questionnaires are administered at baseline (during the diagnostic phase) and evaluate gender identity and dysphoria, body image, quality of life, psychological functioning, and psychiatric comorbidity.

\section{Follow-up and data management}

The short-term follow-up consists of ENIGI visits at baseline and after 3, 6, 9, 12, and 24 months of CHT. We also will perform mid-term follow-up after 10 years of hormonal treatment. In the future we will introduce long-term follow-up visits as well.

Currently, data management is performed by dedicated $\mathrm{PhD}$ students and laboratory technicians. We exchange databases at our twice-yearly research meetings. In the near future, data exchange and storage will be coordinated by a central ENIGI data manager. All ENIGI participants are given an ENIGI research number by which their data are anonymized.

New research proposals are discussed at our ENIGI meetings and all senior research partners have to agree with the proposal. Upon agreement the researcher gets access to the data needed for that specific research question. New ENIGI partners are welcome to join our initiative by contacting one of the senior investigators and filling out a questionnaire that evaluates quality of delivered health care and number of trans persons treated.

\section{RESULTS}

The baseline characteristics of our study population are presented in Table 1. So far 333 trans women and 343 trans men have been included in the ENIGI Endocrine protocol. The mean age at start of follow-up was 30 years and 24 years for trans
Table 1. Characteristics of the study population and cross-sex hormonel treatment regimens

\begin{tabular}{|c|c|c|}
\hline & $\begin{array}{l}\text { Trans } \\
\text { women }\end{array}$ & $\begin{array}{l}\text { Trans } \\
\text { men }\end{array}$ \\
\hline Total number of trans persons ( $\mathrm{n}$ ) & 333 & 343 \\
\hline \multicolumn{3}{|c|}{$\begin{array}{l}\text { Number of trans persons } \\
\text { per center }(n)\end{array}$} \\
\hline $\begin{array}{l}\text { VU University Medical } \\
\text { Centre Amsterdam }\end{array}$ & 178 & 185 \\
\hline Ghent University Hospital & 130 & 69 \\
\hline $\begin{array}{l}\text { Rikshospitalet University } \\
\text { Hospital Oslo }\end{array}$ & 13 & 81 \\
\hline University Hospital Florence & 12 & 8 \\
\hline \multicolumn{3}{|l|}{$\begin{array}{l}\text { Cross-sex hormonal treatment } \\
\text { at start of study (n) }\end{array}$} \\
\hline $\begin{array}{l}\text { Estradiol valerate } \\
\qquad 4 \mathrm{mg} / \text { day }+ \text { CPA }\end{array}$ & 221 & \\
\hline $\begin{array}{l}\text { Estradiol patches } 100 \mathrm{mcg} / \\
24 \text { hours + CPA }\end{array}$ & 109 & \\
\hline Estradiol gel* + CPA & 3 & \\
\hline $\begin{array}{l}\text { Testosterone im } 1000 \mathrm{mg} \\
\text { once per } 12 \text { weeks }\end{array}$ & & 177 \\
\hline $\begin{array}{l}\text { Testosterone im } 250 \mathrm{mg} \\
\text { once per } 2 \text { weeks }\end{array}$ & & 90 \\
\hline Testosterone gel 50 mg/day & & 76 \\
\hline Age, median (range) (y) & $30(16-65)$ & $24(16-51)$ \\
\hline $\begin{array}{l}\text { Systolic blood pressure } \\
\text { (mm Hg) }\end{array}$ & $127(3.3)$ & $122(6.2)$ \\
\hline $\begin{array}{l}\text { Diastolic blood pressure } \\
\text { (mm Hg) }\end{array}$ & $79(3.1)$ & $75(4.5)$ \\
\hline Body weight (kg) & $77(2.8)$ & $71(2.5)$ \\
\hline Length (m) & $1.77(0.02)$ & $1.67(0.02)$ \\
\hline BMI $\left(\mathrm{kg} / \mathrm{m}^{2}\right)$ & $24.4(0.8)$ & $25.3(0.7)$ \\
\hline Current smoking (\%) & 32.7 & 32.1 \\
\hline $\begin{array}{c}\text { Alcohol, median }\left(25^{\text {th }} / 75^{\text {th }}\right. \\
\text { percentile) (units/wk) }\end{array}$ & $0(0-2)$ & $0(0-2)$ \\
\hline
\end{tabular}

$\mathrm{CPA}=$ cyproterone acetate; im = intramuscular.

Data are presented as means (SD) unless stated otherwise.

*Patients treated with estradiol emidrate gel $1 \mathrm{mg}$ twice daily.

women and trans men, respectively. Most trans women are treated with estradiol valerate tablets $(n=221)$, whereas fewer patients are treated with transdermal oestradiol patches $(\mathrm{n}=$ 109) or estradiol gel $(n=3)$. The most frequently prescribed hormonal treatment for trans men is testosterone undecanoate injections $(n=177)$. Ninety trans men are treated with testosterone injections every 2 weeks (Sustanon or Testo-enant) and 76 trans men with testosterone gel. We have complete data on age and treatment at start of follow-up. Missing data is below 7 percent for all other characteristics presented in Table 1.

\section{DISCUSSION}

The ENIGI Endocrine protocol studies the effects and sideeffects of CHT in transgender individuals. Data collection began in 2010 and at time of the first EPATH conference in 
March 2015 in Ghent (Belgium), 333 trans women and 343 trans men were included from 4 different European expertise centers.

Recently, the first results of the ENIGI collaboration were published. Wierckx et al evaluated side-effects and safety of CHT in 53 trans men and 53 trans women. For this study patients from Ghent University Hospital and Rikshospitalet University Hospital Oslo were included. The authors concluded that the treatment modalities used were effective and carried a low risk for side-effects and adverse events at short-time follow-up. ${ }^{15}$ Furthermore, Van Caenegem et al showed that although trans women had a lower bone mineral density and cortical bone size before the start of CHT, their skeletal status was well preserved during the first 2 years of their hormonal treatment, despite substantial muscle loss. ${ }^{16}$ For this study 49 trans women were included from Ghent University Hospital. Additional analyses using the (combined) data of the different centers are ongoing.

To our knowledge, the ENIGI initiative is the first large prospective cohort study to evaluate clinical and side-effects of CHT. In line with the findings of Wierckx et al, previous observational studies showed that CHT of trans men yields a low risk of adverse events in the short and longer term. ${ }^{17,18}$ However, observational studies with longer follow-up times show that the incidence of cardiovascular disease in trans women is higher compared with the control population, whereas this increase is not observed in trans men on testosterone treatment. ${ }^{17}$ Limitations of these studies include the retrospective design (all studies), relative low number of study subjects, ${ }^{19-21}$ and relatively short median duration of follow-up of most of these studies (between 4.4 and 9.0 years). ${ }^{19-23}$ This is of importance, because the largest increase in incidence of lethal cardiovascular events in trans women seems to occur after 10 years of CHT. This was shown by a study by Asscheman et al on total and cause-specific mortality of trans persons receiving hormonal treatment in which 966 trans women and 365 trans men were included with a median duration of followup of 18.5 years. ${ }^{24}$ However, in this study most lethal ischemic cardiac events observed were related to ethinyl estradiol use. This synthetic estrogen is not part of current $\mathrm{CHT}$ protocols since the observation of the increased risk of venous thrombosis related to this compound. ${ }^{22}$ Well-designed prospective studies in large samples are needed to evaluate safety and effectiveness of current hormonal treatment of trans persons.

In ENIGI, all centers use the same study protocol. We use the same standardized questionnaires and use the same devices to measure clinical outcomes. In this way we will be able to include patients from participating centers in combined studies and compare results of the different centers easily. However, the hormonal treatment protocols differ somewhat between participating centers. The most important reason for these discrepancies is that not all treatment modalities are reimbursed in the different European countries. For instance, testosterone undecanoate injections are not covered in the Netherlands. Although these differences were not introduced intentionally, they may provide us with insights into possible different clinical and sideeffects of different treatment options for trans persons. Although we are not performing a randomized controlled trial, important clinical lessons will be learnt.

Up till now we have not collected data on total number of eligible patients, and number and reasons of non-participation. However, in our experience the majority of patients were very motivated to participate in the study and wanted to contribute to improve transgender health care. At our latest research meeting we agreed to start collecting these data from June 2016 onward. Another important improvement is that in the near future data storage and exchange will be coordinated by a central ENIGI data manager. However, due to lack of funding we have not been able to launch a central ENIGI website yet which can be used for data management, exchange of research protocols, and application of new ENIGI partners. Hopefully, with enough resources we will be able to launch this website in the future.

A better understanding and knowledge of clinical and sideeffects of CHT will be essential to optimize hormonal treatment of trans persons. Once a substantial database has been collected, variations in products and dosages may be introduced in the hormonal treatment protocol of trans persons.

Corresponding Author: M.J.H.J. Dekker, MD, PhD, VU University Medical Centre, PO Box 7057, Amsterdam 1007 MB, The Netherlands; E-mail: m.dekker3@vumc.nl

Conflict of Interest: The authors report no conflicts of interest.

Funding: K. Wierckx was supported by the special research funds of Ghent University. E. Van Caenegem is holder of a PhD fellowship from the Research Foundation Flanders. The Department of Endocrinology of the Ghent University Hospital is supported by Bayer for testosterone treatment.

\section{STATEMENT OF AUTHORSHIP}

\section{Category 1}

(a) Conception and Design

K. Wierckx; E. Van Caenegem; M.J.H.J. Dekker; A.D. Fisher; M.A.A. van Trotsenburg; T. Schreiner; B.P. Kreukels; E. Elaut; M. Den Heijer; C. T'Sjoen

(b) Acquisition of Data

K. Wierckx; M.J.H.J. Dekker; E. Van Caenegem; M. Klaver; A.D. Fisher; T. Schreiner; M. Den Heijer; G. T'Sjoen

(c) Analysis and Interpretation of Data M.J.H.J. Dekker; M. Klaver; B.P. Kreukels; E. Elaut; M. Den Heijer; G. T'Sjoen

\section{Category 2}

(a) Drafting the Article

M.J.H.J. Dekker; M. Den Heijer; G. T'Sjoen; A.D. Fisher; T. Schreiner 


\section{(b) Revising It for Intellectual Content All authors}

\section{Category 3}

(a) Final Approval of the Completed Article All authors

\section{REFERENCES}

1. Diagnostic and Statistical Manual of Mental Disorders. 5th Edition. Arlington: American Psychiatric Association; 2015. p. 156-160.

2. Benjamin H. Transsexualism, its nature and therapy. Nervenarzt 1964.

3. Hembree WC, Cohen-Kettenis P, Delemarre-van de Waal HA, et al. Endocrine treatment of transsexual persons: An Endocrine Society clinical practice guideline. J Clin Endocrinol Metab 2009;94:3132-3154.

4. Kreukels BP, Haraldsen IR, De CG, et al. A European network for the investigation of gender incongruence: The ENIGI initiative. Eur Psychiatry 2012;27:445-450.

5. Muhldorfer-Fodor M, Ziegler S, Harms C, et al. Grip force monitoring on the hand: Manugraphy system versus Jamar dynamometer. Arch Orthop Trauma Surg 2014;8:1179-1188.

6. Schmidt RT, Toews JV. Grip strength as measured by the Jamar dynamometer. Arch Phys Med Rehabil 1970;6:321-327.

7. Ferriman D, Gallwey JD. Clinical assessment of body hair growth in women. J Clin Endocrinol Metab 1961;21:1440-1447.

8. Doshi A, Zaheer A, Stiller MJ. A comparison of current acne grading systems and proposal of a novel system. Int $J$ Dermatol 1997;36:416-418.

9. Van Caenegem E, Wierckx K, Taes Y, et al. Bone mass, bone geometry, and body composition in female-to-male transsexual persons after long-term cross-sex hormonal therapy. J Clin Endocrinol Metab 2012;97:2503-2511.

10. Baecke JA, Burema J, Frijters JE. A short questionnaire for the measurement of habitual physical activity in epidemiological studies. Am J Clin Nutr 1982;36:936-942.

11. Dacakis G, Davies S, Oates JM, et al. Development and preliminary evaluation of the transsexual voice questionnaire for male-to-female transsexuals. J Voice 2013;27:312-320.

12. Watson D, Clark LA, Tellegen A. Development and validation of brief measures of positive and negative affect: The PANAS scales. J Pers Soc Psychol 1988;54:1063-1070.
13. Spielberger CD. State-Trait Anger Expression Inventory-2 (STAXI-2); Professional Manual; 1999.

14. Spector IP, Carey MP, Steinberg L. The sexual desire inventory: Development, factor structure, and evidence of reliability. J Sex Marital Ther 1996;22:175-190.

15. Wierckx K, Van CE, Schreiner T, et al. Cross-sex hormone therapy in trans persons is safe and effective at short-time follow-up: Results from the European network for the investigation of gender incongruence. J Sex Med 2014;11:1999-2011.

16. Van CE, Wierckx K, Taes $Y$, et al. Preservation of volumetric bone density and geometry in trans women during cross-sex hormonal therapy: A prospective observational study. Osteoporos Int 2015;26:35-47.

17. Gooren LJ, Wierckx K, Giltay EJ. Cardiovascular disease in transsexual persons treated with cross-sex hormones: Reversal of the traditional sex difference in cardiovascular disease pattern. Eur J Endocrinol 2014;170:809-819.

18. Mueller A, Kiesewetter F, Binder $H$. Long-term administration of testosterone undecanoate every 3 months for testosterone supplementation in female-to-male transsexuals. J Clin Endocrinol Metab 2007;92:3470-3475.

19. Bazarro-Castro M, Sievers C, Fulda S, et al. Comorbidities in transsexual patients under hormonal treatment compared to age- and gender-matched primary care comparison groups. Reprod Syst Sex Disord 2012;1:101. http://dx.doi.org/ 10.4172/2161-038X.1000101.

20. Dhejne $C$, Lichtenstein $P$, Boman $M$, et al. Long-term follow-up of transsexual persons undergoing sex reassignment surgery: Cohort study in Sweden. PLoS One 2011;6:e16885.

21. Wierckx K, Elaut E, Declercq E, et al. Prevalence of cardiovascular disease and cancer during cross-sex hormone therapy in a large cohort of trans persons: A case-control study. Eur J Endocrinol 2013;169:471-478.

22. Asscheman H, Gooren LJ, Eklund PL. Mortality and morbidity in transsexual patients with cross-gender hormone treatment. Metabolism 1989;88:69-73.

23. van Kesteren PJ, Asscheman H, Megens JA, et al. Mortality and morbidity in transsexual subjects treated with cross-sex hormones. Clin Endocrinol (Oxf) 1997;47:337-342.

24. Asscheman H, Giltay EJ, Megens JA, et al. A long-term follow-up study of mortality in transsexuals receiving treatment with cross-sex hormones. Eur J Endocrinol 2011; 164:635-642. 